\title{
Calculation of the Moments and the Moment Generating Function for the Reciprocal Gamma Distribution
}

\author{
By Arne Fransén and Staffan Wrigge
}

\begin{abstract}
In this paper we consider the distribution $G(x)=F^{-1} \int_{0}^{x}(\Gamma(t)){ }^{1} d t$. The aim of the investigation is twofold: first, to find numerical values of characteristics such as moments, variance, skewness, kurtosis, etc.; second, to study analytically and numerically the moment generating function $\varphi(t)=\int_{0}^{\infty} e^{-t x} / \Gamma(x) d x$. Furthermore, we also make a generalization of the reciprocal gamma distribution, and study some of its properties.
\end{abstract}

Introduction. In [4] we considered, among many other things, the distribution $G(x)=F^{-1} \int_{0}^{x}(\Gamma(t))^{-1} d t$, which was suggested as a distribution of possible use in reliability theory by Dr. Gustaf Borenius, the former head of our section. He suggested this distribution because its shape looked like that of the gamma distribution, only with a thinner tail. Moreover, the problem as such was a mathematical challenge. The normalization constant $F$ was calculated and presented to $60 \mathrm{D}$ by Fransén and Wrigge [4], to $80 \mathrm{D}$ by Fransén [3], and to 300D by W. A. Johnson [10].

We were asked by Dr. Samuel Kotz if we could calculate analytically and numerically the moments of the reciprocal gamma distribution. To our surprise, we managed to find not only a useful recurrence relation for the moments $\alpha_{n}=E\left(X^{n}\right)$ but also several analytical expressions.

The aim of this investigation is twofold: first, to find numerical values of characteristics such as moments, variance, skewness, kurtosis, etc.; second, to study analytically and numerically the moment generating function

$$
\varphi(t)=\int_{0}^{\infty}\left(e^{-t x} / \Gamma(x)\right) d x
$$

Furthermore, we also make an obvious generalization of the reciprocal gamma distribution and study some of its properties.

When starting this study we did not know much about the work of our predecessors on similar problems. We learned that Ramanujan [8] as well as Wyman and Wong [17] and Dorning et al. [1] had studied related problems from a purely analytical standpoint. In Erdélyi et al., Vol. 3 [2, pp. 217-224] several functions close to the one studied by us are mentioned. Paley and Wiener used these functions, when studying inversion formulae for the Laplace transform; see [13, p. 39]. We also learned a lot when reading a doctoral thesis from 1887 by A. Lindhagen [11] on the gamma function.

Received September 21, 1982; revised June 20, 1983.

1980 Mathematics Subject Classification. Primary 33A15, 65D20, 65U05; Secondary 44A10, 60E15.

Key words and phrases. Reciprocal gamma distribution, population characteristics, generating function. 
1. A General Formula for the Mean Value $E(\psi(X))$, Where $X$ Has a Generalized Reciprocal Gamma Distribution. We begin with a

Definition. By a generalized reciprocal gamma distribution we mean a distribution with a density function $f(x)=1 / g(x)$, where $g(x)$ satisfies $g(x+1)=x g(x)$ and $x \in[0, \infty)$.

We may then formulate

THEOREM 1. Let $1 / g(x)$ be a generalized reciprocal gamma probability density function, and let $\psi(x)$ be a continuous function such that

$$
E(\psi(X))=\int_{0}^{x} \frac{\psi(x)}{g(x)} d x
$$

exists. Then

$$
E(\psi(X))=\int_{0}^{1} \frac{u F(u)}{g(u+1)} d u
$$

where

$$
\begin{aligned}
F(u) & =\psi(u)+\sum_{k=1}^{\infty} \frac{\psi(u+k)}{\prod_{j=0}^{k-1}(u+j)} \\
& =\psi(u)+\sum_{j=0}^{\infty} \frac{(-1)^{j}}{j !(u+j)}\left(\sum_{k=0}^{\infty} \frac{\psi(u+1+j+k)}{k !}\right)
\end{aligned}
$$

and the series for $u F(u)$ is assumed to converge uniformly in $[0,1]$.

Proof.

$$
\begin{aligned}
E(\psi(X)) & =\sum_{k=0}^{\infty} \int_{k}^{k+1} \frac{\psi(x)}{g(x)} d x=\sum_{k=0}^{\infty} \int_{0}^{1} \frac{\psi(u+k)}{g(u+k)} d u \\
& =\int_{0}^{1} \frac{u F(u)}{g(u+1)} d u .
\end{aligned}
$$

The "partial fraction" is deduced in the ordinary way using the identity

$$
\frac{1}{u(u+1) \cdots(u+n-1)}=\sum_{m=0}^{n-1}\left(\begin{array}{c}
n-1 \\
m
\end{array}\right) \frac{(-1)^{m}}{(n-1) !} \frac{1}{u+m}, \quad n=1,2, \ldots
$$

Of special importance is the case when $\psi(x)$ is periodic with a period equal to one. We may, e.g., determine the constant $c_{2 n}$ such that $c_{2 n} \sin ^{2 n}(\pi x) / \Gamma(x)$ becomes a probability density. (See Section 3b.)

It should be noted that one might as well define the generalized reciprocal gamma distribution demanding that the density function $f(x)$ satisfies $f(x+1)=f(x) / x$.

We have also studied the more general case where the probability density function $f(x)$ satisfies the functional relation

$$
f(x+1)=r(x) f(x)
$$

where $r(x)$ is a rational, or even more general, function. It is then easy to see how to generalize Theorem 1 .

An example of such a function $f(x)$, which we have studied in some detail, is

$$
f(x)=c \prod_{i=1}^{r} \Gamma\left(m_{i} x\right) / \prod_{j=1}^{s} \Gamma\left(n_{j} x\right)
$$


where $m_{i}$ and $n_{j}$ are positive integers, chosen such that the integral $\int_{0}^{\infty} f(x) d x$ exists, and $c$ is a normalization constant. A simple example is

$$
f(x)=c \Gamma(x) / \Gamma(2 x)
$$

giving $r(x)=\frac{1}{2}(2 x+1)^{-1}$.

We plan to present our studies of the more general density functions in (1.1) and (1.2), and corresponding moments and moment generating functions, in a future paper.

\section{Some Formulae for the Moments and the Factorial Moments.}

a. The General Case. Let $X$ be a random variable with the same distribution as in Theorem 1. We want to obtain a useful formula for the moments $\alpha_{n}=E\left(X^{n}\right)$.

Let us define the numbers $\lambda_{n}$ as $\lambda_{n}=\int_{0}^{1}\left(x^{n} / g(x)\right) d x$. We may then formulate

THEOREM 2. Let $\alpha_{n}=\int_{0}^{\infty}\left(x^{n} / g(x)\right) d x$ and $\lambda_{n}=\int_{0}^{1}\left(x^{n} / g(x)\right) d x$. Then the moments $\alpha_{n}$ satisfy the recurrence relation $\alpha_{n}=1+\lambda_{n}-\lambda_{0}+\sum_{k=1}^{n}\left(\begin{array}{l}n \\ k\end{array}\right) \alpha_{k-1}$, with starting value $\alpha_{0}=1$.

Proof.

$$
\alpha_{n}=\int_{0}^{1} \frac{x^{n}}{g(x)} d x+\int_{1}^{\infty} \frac{x^{n}}{g(x)} d x=\lambda_{n}+\int_{0}^{\infty} \frac{(x+1)^{n}}{x g(x)} d x
$$

A simple use of the binomial theorem and some manipulations easily yield Theorem 2.

We may describe the solution of the equation in Theorem 2 in

THEOREM 3. The solution of the recurrence relation in Theorem 2 may be written in the following way

$$
\alpha_{n}=b_{n+1}+\sum_{k=0}^{n} d_{k}(n) \lambda_{k} ; \quad d_{0}(0)=0, \quad d_{n}(n)=1, \quad b_{1}=1,
$$

where $b_{n}$ is the Bell number of order $n$ and the integers $d_{k}(n)$ satisfy

$$
d_{m}(n+1)=\sum_{j=m}^{n}\left(\begin{array}{c}
n+1 \\
j+1
\end{array}\right) d_{m}(j)-\delta_{0 m}, \quad d_{m}(j)=0 \quad \text { for } j<m .
$$

Proof. The numbers $b_{n}$ satisfy the equation

$$
b_{n+1}=\sum_{k=0}^{n}\left(\begin{array}{l}
n \\
k
\end{array}\right) b_{k}, \quad b_{0}=1
$$

(see, e.g., Lunnon, Pleasants and Stephens [12, p. 2]), which is also obtained from the recurrence relation in Theorem 2 . Now, consider

$$
\alpha_{n+1}=1+\lambda_{n+1}-\lambda_{0}+\sum_{j=0}^{n}\left(\begin{array}{c}
n+1 \\
j+1
\end{array}\right)\left\{b_{j+1}+\sum_{k=0}^{j} d_{k}(j) \lambda_{k}\right\} .
$$

Identifying coefficients of $\lambda_{m}$ on both sides of (2.2), we get

$$
d_{m}(n+1)=\sum_{j=m}^{n}\left(\begin{array}{c}
n+1 \\
j+1
\end{array}\right) d_{m}(j)-\delta_{0 m}
$$


The obvious advantage of knowing Theorem 3 is that in order to calculate $E\left(X^{n}\right)$ we only have to know the numbers $\lambda_{n}$. If $1 / g(x)$ may be expanded as $1 / g(x)=$ $\sum_{k=1}^{\infty} c_{k} x^{k}$, then

$$
\lambda_{n}=\sum_{k=1}^{\infty} c_{k} \frac{1}{n+k+1} .
$$

Putting $\psi(x)=(x-1)(x-2) \cdots(x-n)$ in Theorem 1, we may state the following theorem for the factorial moments, viz.

TheOrem 4. Put $\psi(x)=\prod_{i=1}^{n}(x-i)$. Then

$$
E(\psi(X))=\int_{0}^{1} \frac{\sum_{k=0}^{n-1} \prod_{i=1}^{n-k}(x-i)+1}{g(x)} d x+e \int_{0}^{1} \frac{x P(x)}{g(x+1)} d x,
$$

where

$$
\begin{gathered}
P(x)=\sum_{n=0}^{\infty} \frac{(-1)^{n}}{n !(x+n)} \\
e P(x)=\frac{1}{x}+\frac{1}{x(x+1)}+\frac{1}{x(x+1)(x+2)}+\cdots .
\end{gathered}
$$

(Concerning the function $P(x)$ see, e.g., Lindhagen [11, pp. 21-22].)

We note the special case $n=1$, which yields

$$
\alpha_{1}=1+\lambda_{1}+e \int_{0}^{1} \frac{x P(x)}{g(x+1)} d x .
$$

From Theorem 2 we obtain $\alpha_{1}=2+\lambda_{1}-\lambda_{0}$. We conclude that

$$
e \int_{0}^{1} \frac{x P(x)}{g(x+1)} d x=1-\lambda_{0} \text {. }
$$

b. The Special Case. In this case we put

$$
\alpha_{n}=\frac{1}{F} \int_{0}^{\infty} \frac{x^{n}}{\Gamma(x)} d x
$$

and define $\gamma_{n}$ as $\gamma_{n}=F \lambda_{n}$, i.e.,

$$
\gamma_{n}=\int_{0}^{1} \frac{x^{n}}{\Gamma(x)} d x
$$

The recurrence relation in Theorem 2 then becomes

$$
\alpha_{n}=1+\frac{\gamma_{n}-\gamma_{0}}{F}+\sum_{k=1}^{n}\left(\begin{array}{l}
n \\
k
\end{array}\right) \alpha_{k-1} ; \quad \alpha_{0}=1 .
$$

When considering the moment generating function $E\left(e^{-t X}\right)$ the numbers $\beta_{n}=\alpha_{n} / n$ ! are more interesting. Thus we get, from a numerical point of view, the more useful recurrence relation

$$
\beta_{n}=\frac{1}{n !}\left(1+\frac{\gamma_{n}-\gamma_{0}}{F}\right)+\sum_{k=1}^{n} \frac{1}{k(n-k) !} \beta_{k-1}, \quad \beta_{0}=1
$$

To obtain the moments $\alpha_{n}$ we first calculate $\beta_{n}$ using (2.9) and after that we use the identity $\alpha_{n}=n ! \beta_{n}$. 
When calculating the numbers $\gamma_{n}$ we use the expansion

$$
\frac{1}{\Gamma(x)}=\sum_{k=0}^{\infty} a_{k+1} x^{k+1}
$$

The coefficients $a_{k+1}$ converge very fast towards zero and are tabulated by Fransén and Wrigge [4] with addendum by Fransén [3], in both cases to 80D. From (2.4) we get the formula

$$
\gamma_{n}=\sum_{k=0}^{\infty} \frac{a_{k+1}}{n+k+2}, \quad n=0,1,2, \ldots
$$

The values of $\alpha_{n}, \beta_{n}$, and $\gamma_{n}$ are presented in Table I for $n=0,1,2, \ldots, 15$ to 30D.

We also deduced a formula for the coefficients $\alpha_{n}$ using the Euler-Maclaurin summation formula and applied it for checking purposes.

Therefore consider the function

$$
\varphi(t)=F E\left(e^{-t X}\right)=\int_{0}^{\infty} \frac{e^{-t x}}{\Gamma(x)} d x .
$$

The Euler-Maclaurin summation formula with step-length $h=1$ yields ( $B_{2 k}$ are the ordinary Bernoulli numbers)

$$
\varphi(t)=F \sum_{j=0}^{\infty} \frac{(-t)^{j}}{j !} \alpha_{j}=e^{-t+e^{-t}}+\sum_{j=0}^{\infty} \frac{(-t)^{j}}{j !} \sum_{k=[(j+1) / 2]+1}^{\infty} \frac{B_{2 k}}{2 k} a_{2 k-1-j} .
$$

(Cf. Section 3a.)

A remaining problem is how to calculate the coefficients $\delta_{j}$ in the expansion

$$
e^{-t+e^{-t}}=Q(t)=\sum_{j=0}^{\infty} \frac{t^{j}}{j !} \delta_{j}
$$

Differentiating (2.12) with respect to $t$ we get $\dot{Q}(t)=-\left(1+e^{-t}\right) Q(t)$, which after identification of coefficients yields the relation

$$
\delta_{j+1}=-\left(\delta_{j}+(-1)^{j} \sum_{k=0}^{j}(-1)^{k}\left(\begin{array}{l}
j \\
k
\end{array}\right) \delta_{k}\right), \quad \delta_{0}=e .
$$

From (2.11) we get the relation

$$
F \alpha_{j}=(-1)^{j} \delta_{j}+\sum_{k=[(j+1) / 2]+1}^{\infty} \frac{B_{2 k}}{2 k} a_{2 k-1-j}, \quad j=0,1,2, \ldots
$$

Formulae (2.13) and (2.14) were used to check the numerical values of $\alpha_{j}$ calculated by (2.9) for small values of $j(j=0,1,2, \ldots, 6)$.

From Hardy's lectures on Ramanujan [8, p. 196] or from his collected works, Volume IV [9, p. 544], we learn that

$$
\int_{0}^{\infty} \frac{s^{x}}{\Gamma(1+x)} d x=e^{s}-\int_{0}^{\infty} \frac{x^{-1} e^{-s x}}{\pi^{2}+\ln ^{2} x} d x, \quad s>0 .
$$

Differentiating (2.15) $n+1$ times with respect to $s$ and putting $s=1$, we get a formula for the factorial moments

$$
\int_{0}^{\infty} \frac{\prod_{i=1}^{n}(x-i)}{\Gamma(x)} d x=e+(-1)^{n} \int_{0}^{\infty} \frac{x^{n} e^{-x}}{\pi^{2}+\ln ^{2} x} d x
$$


Putting $s=e^{-t}$ in (2.15), we get, after differentiation with respect to $t$, the formula

$$
\varphi(t)=\int_{0}^{\infty} \frac{e^{-t x}}{\Gamma(x)} d x=e^{-t+e^{t}}+\int_{0}^{\infty} \frac{e^{-t-x e}}{\pi^{2}+\ln ^{2} x} d x .
$$

(See also Section 3.)

To obtain an analytical expression for the moments $\alpha_{n}$ in this case, we must first consider a certain generating function, viz.

$$
e^{x e^{-t}}=\sum_{k=0}^{\infty} C_{k}(x)(-1)^{k} \frac{t^{k}}{k !} .
$$

For the functions $C_{k}(x)$ we easily obtain the relations

$$
\begin{aligned}
& C_{k}(x)=\sum_{n=0}^{\infty} \frac{n^{k}}{n !} x^{n}, \\
& C_{k}(x)=x \dot{C}_{k-1}(x), \quad k \geqslant 1, \\
& C_{k}(x)=e^{x} P_{k}(x),
\end{aligned}
$$

where $P_{k}(x)$ is a polynomial of order $k$ with positive integer coefficients,

$$
P_{k}(x)=\sum_{j=0}^{k} S(k, j) x^{j}
$$

$S(k, j)$ denotes Stirling's number of the second kind (Riordan [14, p. 192]),

$$
C_{n}(u+v)=\sum_{k=0}^{n}\left(\begin{array}{l}
n \\
k
\end{array}\right) C_{k}(u) C_{n-k}(v)
$$

Differentiating (2.17) $n$ times with respect to $t$ and putting $t=0$, we obtain

$$
F \alpha_{n}=\sum_{k=0}^{n}\left(\begin{array}{l}
n \\
k
\end{array}\right) C_{k}(1)+\int_{0}^{\infty} \frac{\sum_{k=0}^{n}\left(\begin{array}{l}
n \\
k
\end{array}\right) C_{k}(-x)}{\pi^{2}+\ln ^{2} x} d x .
$$

The value of $C_{k}(1)$ is $e b_{k}$, where $b_{k}$ is the Bell number of order $k$ (see Riordan [14, p. 193]). We note also that the numbers $\delta_{j}$ defined by $(2.12)$ are related to $C_{k}(1)$ in the following way

$$
\delta_{n}=(-1)^{n} \sum_{k=0}^{n}\left(\begin{array}{l}
n \\
k
\end{array}\right) C_{k}(1)=(-1)^{n} e b_{n+1} .
$$

Finally, comparing (2.14) and (2.24), we obtain

$$
\sum_{k=[(n+1) / 2]+1}^{\infty} \frac{B_{2 k}}{2 k} a_{2 k-1-n}=\int_{0}^{\infty} \frac{\sum_{k=0}^{n}\left(\begin{array}{l}
n \\
k
\end{array}\right) C_{k}(-x)}{\pi^{2}+\ln ^{2} x} d x .
$$

Gautschi [5] considered polynomials orthogonal with respect to the reciprocal gamma distribution. Let the polynomials be $\left\{\pi_{k}(x)\right\}$, normalized so that the coefficient of $x^{k}$ equals 1 . Gautschi tabulated the coefficients $\hat{\alpha}_{k}$ and $\hat{\beta}_{k}(k=0(1) 39$, $18 \mathrm{D})$ of the three-term recursion formula

$$
\pi_{k+1}(x)=\left(x-\hat{\alpha}_{k}\right) \pi_{k}(x)-\hat{\beta}_{k} \pi_{k-1}(x) ; \quad \pi_{0}(x)=1, \quad \pi_{-1}(x)=0 .
$$

These polynomials could be used either to calculate or to check the moments of the reciprocal gamma distribution. No discrepancies were found. 
c. Some Population Characteristics in the Special Case. For the sake of completeness only we give the numerical values of the population characteristics mentioned above to 30D. The moments around zero, $\alpha_{n}$, are given in Table I, and from these we get

$$
\begin{aligned}
& \text { mean value }=\alpha_{1}= \\
& 1.934567042147884721183714704369, \\
& \text { variance }=\sigma^{2}=\mu_{2}= \\
& 1.093936334068611315479743413155, \\
& \text { skewness coefficient }=\nu_{1}=\mu_{3} / \sigma^{3}= \\
& 0.815914578635844856917857543121, \\
& \text { coefficient of kurtosis }=\nu_{2}=\mu_{4} / \sigma^{4}-3= \\
& 0.802301598314383564696933612202
\end{aligned}
$$

and the moment-ratios

$$
\begin{array}{ll}
g_{3}=\sqrt{\nu_{1}}= & 0.903279900493664730430818109121 \\
g_{4}=\nu_{2}+3= & 3.802301598314383564696933612202
\end{array}
$$

3. Further Analysis of the Function $\varphi(t)$.

a. Some Analytical Expressions. Consider the function $\varphi(t)=F E\left(e^{-t X}\right)=$ $\int_{0}^{\infty}\left(e^{-t x} / \Gamma(x)\right) d x$. Ramanujan, as mentioned by Hardy in [8], proved the formula

$$
\int_{-\xi}^{\infty} \frac{y^{x}}{\Gamma(1+x)} d x+\int_{0}^{\infty} x^{\xi-1} e^{-y x}\left\{\cos (\pi \xi)-\frac{\sin (\pi \xi)}{\pi} \ln x\right\} \frac{d x}{\pi^{2}+\ln ^{2} x}=e^{y}
$$

when $y>0$ and $\xi \geqslant 0$, using an ingenious method. Putting $\xi=1$, we get

$$
\int_{-1}^{\infty} \frac{y^{x}}{\Gamma(1+x)} d x-\int_{0}^{\infty} \frac{e^{-y x}}{\pi^{2}+\ln ^{2} x} d x=e^{y}
$$

which may be written

$$
\int_{0}^{\infty} \frac{y^{x-1}}{\Gamma(x)} d x=e^{y}+\int_{0}^{\infty} \frac{e^{-y x}}{\pi^{2}+\ln ^{2} x} d x
$$

We proved (3.2) from scratch showing that the Laplace transform of the L.H.S. equals the Laplace transform of the R.H.S. Thereby we used probability, as well as residue, calculus.

Setting $y=e^{-t}$ in (3.2) yields

$$
\varphi(t)=\int_{0}^{\infty} \frac{e^{-t x}}{\Gamma(x)} d x=e^{-t+e^{-t}}+\int_{0}^{\infty} \frac{e^{-t-x e^{-t}}}{\pi^{2}+\ln ^{2} x} d x
$$

We will return to some different forms of Eq. (3.3) in Section 3c.

Several interesting analytical expressions of $\varphi(t)$ may be obtained using the Euler-Maclaurin summation formula. Thus we calculate

$$
\varphi_{1}(t)=\sum_{k=1}^{\infty} \frac{e^{-t k}}{\Gamma(k)}=e^{-t+e^{-t}}
$$

and

$$
\varphi_{2}(t)=\sum_{k=0}^{\infty} \frac{e^{-t(k+1 / 2)}}{\Gamma\left(k+\frac{1}{2}\right)}=\frac{e^{-t / 2}}{\sqrt{\pi}}+\left(2 N\left(2^{1 / 2} e^{-t / 2}\right)-1\right) e^{-t+e^{-t}}
$$


where $N(\cdot)$ denotes the standardized normal distribution function, i.e.

$$
N(t)=\frac{1}{\sqrt{2 \pi}} \int_{-\infty}^{t} e^{-x^{2} / 2} d x
$$

A formal use of the summation formula yields the equations

$$
\varphi(t)=e^{-t+e^{-t}}+\sum_{j=0}^{\infty}(-1)^{j} \frac{t^{j}}{j !} \sum_{k=[(j+1) / 2]+1}^{\infty} \frac{B_{2 k}}{2 k} a_{2 k-1-j}
$$

and

$$
\varphi(t)=\frac{1}{2}\left(\varphi_{1}(t)+\varphi_{2}(t)\right)+\sum_{j=0}^{\infty} \frac{(-t)^{j}}{j !} \sum_{k=[(j+1) / 2]+1}^{\infty} \frac{B_{2 k}}{2 k}\left(\frac{1}{2}\right)^{2 k} a_{2 k-1-j} .
$$

(Cf. Section 2b.) The more complicated case with the step-length $h=\frac{1}{4}$ is treated in a short note (Wrigge [16]).

We may generalize these results in

THEOREM 5. Let $f(x)=1 / g(x)$ be a generalized reciprocal gamma probability density function such that $f(0)=0, f(0)$ is finite and nonzero and suppose that $1 / g(x)=\sum_{k=1}^{\infty} c_{k} x^{k}$. Define $\kappa(t)=E\left(e^{-t X}\right)$. Then formally we have, if $f^{(n)}(\infty)=0$ for $n=0,1,2, \ldots$,

$$
\kappa(t)=\kappa_{1}(t)+\sum_{j=0}^{\infty} \frac{(-t)^{j}}{j !} \sum_{k=[(j+1) / 2]+1}^{\infty} \frac{B_{2 k}}{2 k} c_{2 k-1-j}
$$

and

$$
\kappa(t)=\frac{1}{2}\left(\kappa_{1}(t)+\kappa_{2}(t)\right)+\sum_{j=0}^{\infty} \frac{(-t)^{j}}{j !} \sum_{k=[(j+1) / 2]+1}^{\infty} \frac{B_{2 k}}{2 k}\left(\frac{1}{2}\right)^{2 k} c_{2 k-1-j},
$$

where

$$
\kappa_{1}(t)=\frac{e^{-t+e^{-t}}}{g(1)} \text { and } \kappa_{2}(t)=\frac{e^{-t / 2}}{g\left(\frac{1}{2}\right)}+\frac{\sqrt{\pi}}{g\left(\frac{1}{2}\right)}\left(2 N\left(2^{1 / 2} e^{-t / 2}\right)-1\right) e^{-t+e^{-t}} .
$$

Using Theorem 1, we may establish the following result.

THEOREM 6. Let $f(x)=1 / g(x)$ be a generalized reciprocal gamma probability density function. Define $\kappa(t)=E\left(e^{-t X}\right)$. Then

$$
\kappa(t)=\int_{0}^{1} \frac{e^{-t u} F_{1}\left(1 ; u ; e^{-t}\right)}{g(u)} d u,
$$

where ${ }_{1} F_{1}(\alpha ; \gamma ; x)$ denotes the confluent hypergeometric function (see Sneddon [15, $p$. 35]). An alternative expression is

$$
\kappa(t)=\int_{0}^{1} \frac{e^{-t u}}{g(u)} d u+e^{-t+e^{-t}} \int_{0}^{1} \frac{e^{-t u}}{g(u+1)} d u+e^{-t+e^{-t}} \int_{0}^{1} \frac{e^{-t u}}{g(u)} G(u) d u,
$$

where

$$
G(u)=\sum_{k=1}^{\infty} \frac{(-1)^{k} e^{-t k}}{k !(u+k)}
$$


The function $\varphi(t)$ is related to the beta function in a natural way. Consider the well-known identity

$$
\int_{0}^{1} t^{x-1}(1-t)^{y-1} d t=\frac{\Gamma(x) \Gamma(y)}{\Gamma(x+y)} .
$$

We divide both sides by $\Gamma(x) \Gamma(y)$, multiply by $e^{-s(x+y)}$ and integrate with respect to $x$ and $y$ between 0 and $\infty$. Formally we get

$$
\int_{0}^{1} \frac{\varphi(s-\ln (t)) \varphi(s-\ln (1-t))}{t(1-t)} d t=\int_{0}^{\infty} \int_{0}^{\infty} \frac{e^{-s(x+y)}}{\Gamma(x+y)} d x d y=-\dot{\varphi}(s) .
$$

The last part of (3.9) will be proved using Eq. (3.12).

We will prove that

$$
\varphi(t)=\int_{0}^{\infty} \int_{0}^{\infty} \frac{e^{-t(x+y)}}{\Gamma(x+y+1)} d x d y .
$$

Therefore consider the auxiliary function

$$
G_{t}(s)=\iint_{x+y \leqslant s ; x, y \geqslant 0} \frac{e^{-t(x+y)}}{\Gamma(x+y+1)} d x d y
$$

Using the mean value theorem of integral calculus, we get

$$
G_{t}(s+\Delta s)-G_{t}(s)=\frac{e^{-t(s+\theta \Delta s)}}{\Gamma(s+\theta \Delta s+1)} \int_{s \leqslant x+y \leqslant s+\Delta s} d x d y ; \quad \theta \in[0,1] .
$$

This easily yields

$$
\frac{d}{d s} G_{t}(s)=\frac{s e^{-t s}}{\Gamma(s+1)}=\frac{e^{-t s}}{\Gamma(s)}
$$

We finally get

$$
G_{t}(\infty)=\varphi(t)=\int_{0}^{\infty} \frac{e^{-t x}}{\Gamma(x)} d x=\int_{0}^{\infty} \int_{0}^{\infty} \frac{e^{-t(x+y)}}{\Gamma(x+y+1)} d x d y
$$

The last part of (3.9) may now be proved using similar methods or simply by differentiating (3.10) with respect to $t$.

b. Some Interesting Inequalities. We start with Weierstrass' formula for $1 / \Gamma(x)$, i.e.

$$
\frac{1}{\Gamma(x)}=e^{\gamma x} x \prod_{n=1}^{\infty}\left(1+\frac{x}{n}\right) e^{-x / n} .
$$

However, since $(1+x / n) e^{-x / n} \leqslant 1$, we get

$$
\frac{1}{\Gamma(x)} \leqslant e^{-x v_{k}} \sum_{j=0}^{k} c_{j, k} x^{j+1}, \quad \text { where } v_{k}=\sum_{n=1}^{k} n^{-1}-\gamma,
$$

and the numbers $c_{j, k}$ are related to the Stirling numbers of the first kind, viz., $c_{j, k}=(s(k+1, j+1) / k !)(-1)^{j+k}$. (See e.g. Riordan [14, p. 90].) This yields the estimate

$$
\varphi(t) \leqslant \sum_{j=0}^{k} c_{j, k} \frac{(j+1) !}{\left(t+v_{k}\right)^{j+2}} .
$$

In Table II we give the values of the upper limit for $k=24$ and $t=0.0(0.1) 5.0$. 
Another inequality for $\varphi(t)$ may be obtained using the multiplication theorem for the gamma function. We see that

$$
\varphi(t)=n \int_{0}^{\infty} \frac{e^{-n t x}}{\Gamma(n x)} d x, \quad n=1,2,3, \ldots
$$

But it is known that $\Gamma(n x)=(2 \pi)^{(1-n) / 2} n^{n x-1 / 2} \Gamma(x) \prod_{k=1}^{n-1} \Gamma(x+k / n)$. This yields

$$
\varphi(t)=n^{3 / 2} \int_{0}^{\infty} \frac{e^{-x(n t+n \log n)}}{(2 \pi)^{(1-n) / 2} \Gamma(x) \prod_{k=1}^{n-1} \Gamma(x+k / n)} d x .
$$

Putting $\Gamma\left(x_{0}\right)=\min _{x>0} \Gamma(x)$ and $c=\Gamma\left(x_{0}\right) / \sqrt{2 \pi}$, we may write

$$
\varphi(n t+n \log n) \geqslant \frac{c^{n-1}}{n^{3 / 2}} \varphi(t), \quad n=1,2,3, \ldots
$$

Similar inequalities may be deduced for the more general transformation

$$
g(a, t)=\int_{0}^{\infty} \frac{e^{-a x} f(t x)}{\Gamma(x)} d x .
$$

Before continuing the analysis of $\varphi(t)$ we note the obvious inequality (from (3.3)),

$$
\varphi(t) \geqslant e^{-t+e^{-t}} \text {. }
$$

We will now deduce a different lower limit for $\varphi(t)$ using a variant of Jensen's inequality, viz., $\exp \left(\int_{u}^{u+1} \ln (f(x)) d x\right) \leqslant \int_{u}^{u+1} f(x) d x$, where $f(x)$ is supposed to be continuous and positive.

We put

$$
G(t, u)=\int_{u}^{u+1} \ln \frac{e^{-t x}}{\Gamma(x)} d x
$$

and get

$$
G(t, u)=-t\left(u+\frac{1}{2}\right)-u \ln u+u-\ln \sqrt{2 \pi}
$$

But

$$
e^{G(t, u)} \leqslant \int_{u}^{u+1} \frac{e^{-t x}}{\Gamma(x)} d x
$$

Summing over $u=0,1,2, \ldots$, we obtain

$$
\varphi(t) \geqslant \frac{e^{-t / 2}}{\sqrt{2 \pi}}\left(1+\sum_{k=1}^{\infty} \frac{e^{k(1-t)}}{k^{k}}\right) .
$$

The simpler inequality (3.18) is slightly better in the interval $(0,0.6)$, see Table III.

We may easily generalize the result in (3.20), viz.,

THEOREM 7. Let $f(x)=1 / g(x)$ be a generalized reciprocal gamma probability density function. Define $\kappa(t)=E\left(e^{-t X}\right)$. Then

$$
\kappa(t) \geqslant K e^{-t / 2}\left(1+\sum_{k=1}^{\infty} \frac{e^{k(1-t)}}{k^{k}}\right),
$$

where $K=e^{-\int_{0}^{1} \ln (g(x)) d x}$. 
An interesting example, which includes (3.20) as a special case, is given by the density function

$$
f_{2 n}(x)=c_{2 n} \frac{\sin ^{2 n}(\pi x)}{\Gamma(x)}
$$

$\left(c_{2 n}\right.$ is a normalization constant and $\left.c_{0}=1 / F\right), n=0,1,2, \ldots$ Define $\kappa_{2 n}(t)=$ $E\left(e^{-t X}\right)$ in this case. Then

$$
\kappa_{2 n}(t) \geqslant c_{2 n} \frac{e^{-t / 2}}{2^{2 n} \sqrt{2 \pi}}\left(1+\sum_{k=1}^{\infty} \frac{e^{k(1-t)}}{k^{k}}\right) .
$$

Proof. Simply note that $\int_{0}^{1} \ln (\sin (\pi x)) d x=-\ln 2$.

c. Numerical Calculation of $\varphi(t)$. We set ourselves the task to compute $\varphi(t)$ to 6D for $t=0.0(0.1) 5.0$. In all we considered more than 10 different methods to compute $\varphi(t)$ to the required accuracy. The methods used could roughly be divided into Gaussian quadrature rules and series expansions.

For small values of $t$ there are several useful expansions. We may, e.g., consider

$$
\varphi(t)=\int_{0}^{1} \frac{e^{-t x}}{\Gamma(x)} d x+e^{-t} \int_{0}^{\infty} \frac{e^{-t x}}{x \Gamma(x)} d x
$$

from which we get the expansion (cf. Section $2 b$ )

$$
\varphi(t)=\sum_{n=0}^{\infty} \frac{(-1)^{n} t^{n}}{n !} \gamma_{n}+e^{-t}\left(F-\gamma_{0}\right)+F e^{-t} \sum_{n=1}^{\infty} \frac{(-1)^{n} t^{n}}{n} \beta_{n-1} .
$$

More rewarding from a computational point of view was to use the Euler-Maclaurin expansions with step-lengths $h=1$ resp. $h=\frac{1}{2}$ (formulae (3.6) and (3.7)). These expansions may in this case be regarded as the Taylor series expansions in disguise. Thus, (3.6) gave $\varphi(t)$ correct to $6 \mathrm{D}$ in the interval $[0,2.0]$. Note that the somewhat complicated coefficients which occur in (3.6) may be calculated from or checked against (2.14). Equation (3.7) gave $\varphi(t)$ correct to 6D in the entire interval $[0,5.0]$. The coefficients in (3.7) were truncated with an upper limit equal to 30 and calculated using high-precision techniques. (Note that we only know the numerical values of $a_{1}, a_{2}, \ldots, a_{61}$; see [3] and [4].) The achieved numerical results of $\varphi(t)$ using the Euler-Maclaurin methods are presented in Table IV.

For larger values of $t$ it is useful to use Watson's Lemma. Applied to $\varphi(t)$, the lemma yields

$$
\varphi(t) \sim \sum_{n=2}^{\infty} \frac{a_{n-1}(n-1) !}{t^{n}}, \quad \text { where } \frac{1}{\Gamma(x)}=\sum_{n=2}^{\infty} a_{n-1} x^{n-1} .
$$

Values of $\varphi(t)$ correct to 6D were obtained from $t=3.8$.

Finally, we turn our attention to the Gaussian quadrature rules. To be able to use a Gauss-Laguerre quadrature rule we rewrite (3.3) in the following two ways, viz.

$$
\varphi(t)=e^{-t+e^{-t}}+\int_{0}^{\infty} \frac{e^{-u}}{\pi^{2}+(t+\ln (u))^{2}} d u
$$

and

$$
\varphi(t)=e^{-t+e^{-t}}+\int_{0}^{\infty} \frac{e^{-z+e^{-z}}}{\pi^{2}+(t-z)^{2}} d z+\int_{0}^{\infty} \frac{e^{-z} e^{2 z-e^{z}}}{\pi^{2}+(t+z)^{2}} d z
$$


We evaluated numerically the integrals occurring in (3.24) and (3.25) using GaussLaguerre quadrature rules. However, none of the formulae were sufficient to yield 6D even when using a 15-point formula. The best result was achieved using (3.25) and a 15-point formula. The maximum absolute error was then about $4 \times 10^{-4}$ in the required interval.

We considered the Gauss-Christoffel quadrature rule with respect to the weight distribution $w(x)=1 / \Gamma(x)$ on [ $0, \infty)$, which is also mentioned by Gautschi [5]. In Table $\mathrm{V}$ we give weights $p_{i}(n)$ and abscissae $x_{i}(n)$ together with the remainder term coefficients $c_{n}$ for $n=14,15$. Thus we get

$$
\int_{0}^{\infty} \frac{f(x)}{\Gamma(x)} d x=\sum_{i=1}^{n} p_{i}(n) f\left(x_{i}(n)\right)+c_{n} f^{(2 n)}(\xi), \quad 0<\xi<\infty .
$$

When calculating the weights and abscissae we used the well-known methods presented by Golub and Welsch [6] and Gustafson [7].

Using (3.26) with $f(x)=e^{-t x}, n=14$ and 15, and comparing the results with each other (and with other results), we could determine $\varphi(t)$ to $6 \mathrm{D}$ in the required interval. The numerical result for $n=15$ appears in Table VI.

Note that the data in Table VI are subject to a progressively increasing error (beginning with $\varphi(2.8)$ ) so that the final entry is correct only to $6 \mathrm{D}$. On the other hand, comparing Table IV with Table I in [16], we see that Table IV is correct to $10 \mathrm{D}$.

The discrepancies between Table IV and Table VI may be interpreted in the following way. The function $\varphi(t)$ does not behave like an exponential polynomial for large values of the argument $t$. When using (3.26) with $f(x)=e^{-t x}$, we in fact put

$$
\varphi(t) \approx \sum_{i=1}^{n} p_{i}(n) e^{-t x_{i}(n)}
$$

and it is obvious from a comparison of Table IV and Table VI that this approximation is good only for "small" values of $t$. Therefore we think it is valuable to give Table VI with 10 decimals even if only 6 are correct throughout.

4. Tables. In this section we present the tables previously mentioned, i.e.,

I. The values of

$$
\alpha_{n}=\frac{1}{F} \int_{0}^{\infty} \frac{x^{n}}{\Gamma(x)} d x, \quad \beta_{n}=\frac{\alpha_{n}}{n !}, \quad \gamma_{n}=\int_{0}^{1} \frac{x^{n}}{\Gamma(x)} d x,
$$

for $n=0,1,2, \ldots, 15$ to $30 \mathrm{D}$.

II. Tabulation of the upper limit

$$
\mathrm{UL}_{k}(t)=\sum_{j=0}^{k} c_{j, k} \frac{(j+1) !}{\left(t+v_{k}\right)^{j+2}}
$$

of $\varphi(t)$ for $k=24$ and $t=0.0(0.1) 5.0((3.15))$.

III. Tabulation of the lower limits

$$
\mathrm{LL}_{1}(t)=e^{-t+e^{-t}} \text { and } \mathrm{LL}_{2}(t)=\frac{e^{-t / 2}}{\sqrt{2 \pi}}\left(1+\sum_{k=1}^{\infty} \frac{e^{k(1-t)}}{k^{k}}\right)
$$

of $\varphi(t)$ for $t=0.0(0.1) 5.0((3.18)$ and (3.20)). 
TABLE I

Values of $\alpha_{n}, \beta_{n}$ and $\gamma_{n}$ to 30D for $n$ up to 15

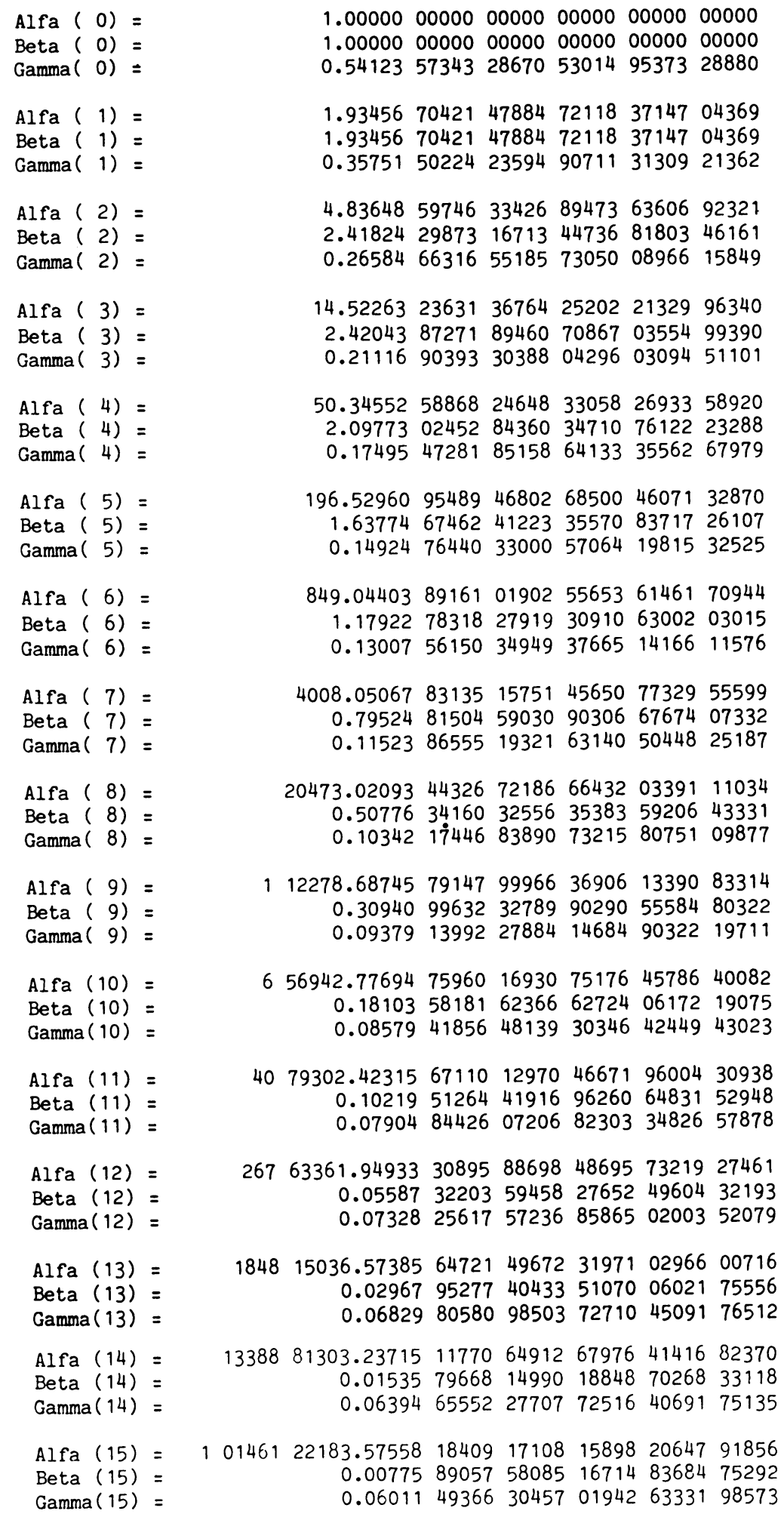


TABLE II

Upper limit of $\varphi(t)$ to $6 \mathrm{D}$

$\begin{array}{cccccc}t & \mathrm{UL}_{24} & t & \mathrm{UL}_{24} & t & \mathrm{UL}_{24} \\ 0.0 & 3.104091 & 1.7 & 0.307953 & 3.4 & 0.092232 \\ 0.1 & 2.545533 & 1.8 & 0.281396 & 3.5 & 0.087315 \\ 0.2 & 2.111334 & 1.9 & 0.257935 & 3.6 & 0.082767 \\ 0.3 & 1.769449 & 2.0 & 0.237128 & 3.7 & 0.078553 \\ 0.4 & 1.497054 & 2.1 & 0.218605 & 3.8 & 0.074642 \\ 0.5 & 1.277650 & 2.2 & 0.202057 & 3.9 & 0.071006 \\ 0.6 & 1.099143 & 2.3 & 0.187224 & 4.0 & 0.067621 \\ 0.7 & 0.952553 & 2.4 & 0.173885 & 4.1 & 0.064465 \\ 0.8 & 0.831128 & 2.5 & 0.161855 & 4.2 & 0.061519 \\ 0.9 & 0.729740 & 2.6 & 0.150973 & 4.3 & 0.058764 \\ 1.0 & 0.644448 & 2.7 & 0.141103 & 4.4 & 0.056184 \\ 1.1 & 0.572196 & 2.8 & 0.132128 & 4.5 & 0.053766 \\ 1.2 & 0.510592 & 2.9 & 0.123946 & 4.6 & 0.051497 \\ 1.3 & 0.457746 & 3.0 & 0.116470 & 4.7 & 0.049364 \\ 1.4 & 0.412154 & 3.1 & 0.109623 & 4.8 & 0.047358 \\ 1.5 & 0.372611 & 3.2 & 0.103338 & 4.9 & 0.045468 \\ 1.6 & 0.338142 & 3.3 & 0.097559 & 5.0 & 0.043687\end{array}$

TABLE III

Lower limits of $\varphi(t)$ to 6D

$\begin{array}{ccc}t & L_{1} & L_{2} \\ 0.0 & 2.718282 & 2.625200 \\ 0.1 & 2.236333 & 2.163212 \\ 0.2 & 1.856570 & 1.800680 \\ 0.3 & 1.553978 & 1.513054 \\ 0.4 & 1.310384 & 1.282521 \\ 0.5 & 1.112412 & 1.095987 \\ 0.6 & 0.950100 & 0.943715 \\ 0.7 & 0.815940 & 0.818377 \\ 0.8 & 0.704215 & 0.714406 \\ 0.9 & 0.610528 & 0.627526 \\ 1.0 & 0.531464 & 0.554424 \\ 1.1 & 0.464344 & 0.492513 \\ 1.2 & 0.407055 & 0.439753 \\ 1.3 & 0.357912 & 0.394525 \\ 1.4 & 0.315561 & 0.355534 \\ 1.5 & 0.278909 & 0.321739 \\ 1.6 & 0.247065 & 0.292296\end{array}$

$\begin{array}{ccc}t & L_{1} & L_{2} \\ 1.7 & 0.219300 & 0.266516 \\ 1.8 & 0.195011 & 0.243837 \\ 1.9 & 0.173699 & 0.223793 \\ 2.0 & 0.154948 & 0.206001 \\ 2.1 & 0.138409 & 0.190140 \\ 2.2 & 0.123787 & 0.175944 \\ 2.3 & 0.110832 & 0.163189 \\ 2.4 & 0.099333 & 0.151685 \\ 2.5 & 0.089107 & 0.141273 \\ 2.6 & 0.080000 & 0.131817 \\ 2.7 & 0.071877 & 0.123202 \\ 2.8 & 0.064623 & 0.115328 \\ 2.9 & 0.058136 & 0.108112 \\ 3.0 & 0.052329 & 0.101479 \\ 3.1 & 0.047125 & 0.095367 \\ 3.2 & 0.042458 & 0.089721 \\ 3.3 & 0.038269 & 0.084494\end{array}$

$\begin{array}{ccc}\mathrm{t} & \mathrm{LL}_{1} & \mathrm{LL}_{2} \\ 3.4 & 0.034506 & 0.079644 \\ 3.5 & 0.031123 & 0.075135 \\ 3.6 & 0.028081 & 0.070935 \\ 3.7 & 0.025342 & 0.067016 \\ 3.8 & 0.022877 & 0.063353 \\ 3.9 & 0.020656 & 0.059926 \\ 4.0 & 0.018654 & 0.056713 \\ 4.1 & 0.016850 & 0.053698 \\ 4.2 & 0.015222 & 0.050865 \\ 4.3 & 0.013754 & 0.048200 \\ 4.4 & 0.012429 & 0.045692 \\ 4.5 & 0.011233 & 0.043328 \\ 4.6 & 0.010153 & 0.041098 \\ 4.7 & 0.009178 & 0.038993 \\ 4.8 & 0.008298 & 0.037005 \\ 4.9 & 0.007502 & 0.035127 \\ 5.0 & 0.006784 & 0.033350\end{array}$

TABLE IV

Values of $\varphi(t)$ to 10D by the Euler-Maclaurin formula

$\begin{array}{cccccc}\mathrm{t} & \varphi(\mathrm{t}) & \mathrm{t} & \varphi(\mathrm{t}) & \mathrm{t} & \varphi(\mathrm{t}) \\ 0.0 & 2.8077702420 & 1.7 & 0.3009335120 & 3.4 & 0.0914210330 \\ 0.1 & 2.3262370474 & 1.8 & 0.2753948016 & 3.5 & 0.0865816184 \\ 0.2 & 1.9467718218 & 1.9 & 0.2527806955 & 3.6 & 0.0821024199 \\ 0.3 & 1.6443584989 & 2.0 & 0.2326807977 & 3.7 & 0.0779495893 \\ 0.4 & 1.4008236962 & 2.1 & 0.2147517803 & 3.8 & 0.0740930688 \\ 0.5 & 1.2027934333 & 2.2 & 0.1987050126 & 3.9 & 0.0705061033 \\ 0.6 & 1.0403059617 & 2.3 & 0.1842967120 & 4.0 & 0.0671648226 \\ 0.7 & 0.9058566158 & 2.4 & 0.1713200562 & 4.1 & 0.0640478828 \\ 0.8 & 0.7937313323 & 2.5 & 0.1595988329 & 4.2 & 0.0611361584 \\ 0.9 & 0.6995357300 & 2.6 & 0.1489822986 & 4.3 & 0.0584124762 \\ 1.0 & 0.6198584141 & 2.7 & 0.1393409958 & 4.4 & 0.0558613851 \\ 1.1 & 0.5520275472 & 2.8 & 0.1305633342 & 4.5 & 0.0534689567 \\ 1.2 & 0.4939329844 & 2.9 & 0.1225527820 & 4.6 & 0.0512226118 \\ 1.3 & 0.4438950131 & 3.0 & 0.1152255491 & 4.7 & 0.0491109691 \\ 1.4 & 0.4005665647 & 3.1 & 0.1085086674 & 4.8 & 0.0471237134 \\ 1.5 & 0.3628597079 & 3.2 & 0.1023383935 & 4.9 & 0.0452514796 \\ 1.6 & 0.3298899227 & 3.3 & 0.0966588753 & 5.0 & 0.0434857514\end{array}$




\section{TABLE V}

Abscissae $x_{i}(n)$ and weights $p_{i}(n)$ to $25 \mathrm{~S}$ for $n=14$ and 15

$n$

14 i

$1 \quad 1.622858744052096654480454 \mathrm{E}-01$

$2 \quad 5.229208541663484816942969 \mathrm{E}-01$

$31.051449279617592254069644 \mathrm{E}+00$

$4 \quad 1.726854794707082013337275 \mathrm{E}+00$

$5 \quad 2.537139970455747796982883 E+00$

$6 \quad 3.477139770547848558819249 \mathrm{E}+00$

$74.547183105381307655844180 \mathrm{E}+00$

$85.752809248184422223580066 \mathrm{E}+00$

$97.105517354977053201482566 \mathrm{E}+00$

$108.624916011709722666870543 \mathrm{E}+00$

$11 \quad 1.034350430938327785568881 \mathrm{E}+01$

$121.231779194476028260112930 \mathrm{E}+01$

$131.465920463327447787732363 \mathrm{E}+01$

$141.765918673901601163881919 \mathrm{E}+01$

$1.490070588640254308122436 \mathrm{E}-01$

$4.819351400658091188356546 \mathrm{E}-01$

$9.723272335870747628473832 \mathrm{E}-01$

$1.601001667819464486376536 \mathrm{E}+00$

2. $356286743334194675877424 \mathrm{E}+00$

$3.232350782165203111136036 \mathrm{E}+00$

4.227983287203939526165386E+00

$5.346137300427159830530296 \mathrm{E}+00$

$6.594175177757317293887338 \mathrm{E}+00$

$7.984927181287134330907394 \mathrm{E}+00$

$9.539032836474949476943656 \mathrm{E}+00$

$1.128985951391608900789341 \mathrm{E}+01$

$1.329475808401580688647499 \mathrm{E}+01$

$1.566620236805261061598493 E+01$

$1.869771150641537821275855 \mathrm{E}+01$ $p_{i}$

4.666936308723003950950625E-02 $2.646109639584808299341717 \mathrm{E}-01$ $6.215442051821438630110274 \mathrm{E}-01$ $8.142350693171694265817131 \mathrm{E}-01$ $6.414191022297807547724504 \mathrm{E}-01$ $3.099598643455755089011735 \mathrm{E}-01$ 9.148075324998564823913511E-02 $1.612928241047227855479475 \mathrm{E}-02$ $1.630797462009361385772756 \mathrm{E}-03$ $8.849849557915662269500680 \mathrm{E}-05$ $2.317750836324959203635878 \mathrm{E}-06$ $2.446556157452933003045058 \mathrm{E}-08$ $7.366805939154725353566559 \mathrm{E}-11$ $2.653882898025302661903184 \mathrm{E}-14$

3.924675165725691451042447E-02 2.260891662923499176678823E-01 $5.527923916882637016023382 \mathrm{E}-01$ $7.759201771782941429763990 \mathrm{E}-01$ $6.757927480583987655263235 \mathrm{E}-01$ 3. $736746571000929295131507 \mathrm{E}-01$ $1.312282470710664383753163 \mathrm{E}-01$ $2.885258016254480346810935 \mathrm{E}-02$ $3.860321080491039705432555 \mathrm{E}-03$ $3.002822090665240753851297 \mathrm{E}-04$ $1.265843407897613382797982 E-05$ $2.589555120399712100012903 E-07$ 2. $136113605313739301854631 \mathrm{E}-09$ 4. $988209679385246841957279 \mathrm{E}-12$ $1.356702577187496841722868 \mathrm{E}-15$

$c_{15}=1.3723708 \mathrm{E}-17$

TABLE VI

Values of $\varphi(t)$ to 10D by Gaussian quadrature rule

$\begin{array}{cc}\mathrm{t} & \varphi(\mathrm{t}) \\ & \\ 0.0 & 2.8077702420 \\ 0.1 & 2.3262370474 \\ 0.2 & 1.9467718218 \\ 0.3 & 1.6443584989 \\ 0.4 & 1.4008236962 \\ 0.5 & 1.2027934333 \\ 0.6 & 1.0403059617 \\ 0.7 & 0.9058566158 \\ 0.8 & 0.7937313323 \\ 0.9 & 0.6995357300 \\ 1.0 & 0.6198584141 \\ 1.1 & 0.5520275472 \\ 1.2 & 0.4939329844 \\ 1.3 & 0.4438950131 \\ 1.4 & 0.4005665647 \\ 1.5 & 0.3628597079 \\ 1.6 & 0.3298899227\end{array}$

$\mathrm{t} \varphi(\mathrm{t})$

$\begin{array}{ll}1.7 & 0.3009335120\end{array}$

$\begin{array}{ll}1.8 & 0.2753948016\end{array}$

$\begin{array}{ll}1.9 & 0.2527806955\end{array}$

$\begin{array}{ll}2.0 & 0.2326807977\end{array}$

$\begin{array}{ll}2.1 & 0.2147517803\end{array}$

$\begin{array}{ll}2.2 & 0.1987050126\end{array}$

$2.3 \quad 0.1842967120$

$2.4 \quad 0.1713200562$

$\begin{array}{ll}2.5 & 0.1595988329\end{array}$

$2.6 \quad 0.1489822986$

$\begin{array}{ll}2.7 & 0.1393409958\end{array}$

$2.8 \quad 0.1305633341$

$\begin{array}{ll}2.9 & 0.1225527819\end{array}$

$3.0 \quad 0.1152255490$

3.10 .1085086671

3.20 .1023383931

$\begin{array}{ll}3.3 & 0.0966588746\end{array}$ $\mathrm{t} \quad \varphi(\mathrm{t})$

$\begin{array}{ll}3.4 & 0.0914210319\end{array}$

$3.5 \quad 0.0865816168$

$3.6 \quad 0.0821024175$

$\begin{array}{ll}3.7 & 0.0779495857\end{array}$

$\begin{array}{ll}3.8 & 0.0740930635\end{array}$

3.90 .0705060958

$\begin{array}{ll}4.0 & 0.0671648119\end{array}$

$\begin{array}{ll}4.1 & 0.0640478678\end{array}$

$\begin{array}{ll}4.2 & 0.0611361378\end{array}$

$\begin{array}{ll}4.3 & 0.0584124481\end{array}$

$\begin{array}{ll}4.4 & 0.0558613473\end{array}$

$\begin{array}{ll}4.5 & 0.0534689064\end{array}$

$\begin{array}{ll}4.6 & 0.0512225456\end{array}$

$\begin{array}{ll}4.7 & 0.0491108830\end{array}$

$\begin{array}{ll}4.8 & 0.0471236023\end{array}$

4.90 .0452513376

$5.0 \quad 0.0434855716$ 
IV. $\varphi(t)=\int_{0}^{\infty}\left(e^{-t x} / \Gamma(x)\right) d x$ tabulated to 10D for $t=0.0(0.1) 5.0$ by means of the Euler-Maclaurin expansion in (3.7).

V. The values of the numbers $p_{i}(n)$ and $x_{i}(n)$ corresponding to the weight function $1 / \Gamma(x)($ cf. $(3.26)), n=14$ and 15 .

VI. $\varphi(t)$ tabulated to $10 \mathrm{D}$ for $t=0.0(0.1) 5.0$ by means of Gaussian quadrature (cf. (3.26)).

Comparing the values of Tables IV and VI, we thus may rely on all of them up to 6D.

Acknowledgment. We want to thank Dr. Jacques Dutka, who gave us several very valuable references and comments.

National Defence Research Institute

Division I, Section 123

S-102 54 Stockholm, Sweden

1. J. J. Dorning, B. Nicolaenko \& J. K. ThURber, "An integral identity due to Ramanujan which occurs in neutron transport theory," J. Math. Mech., v. 19, No. 5, 1969, pp. 429-438.

2. A. Erdélyi, W. Magnus, F. Oberhettinger \& F. G. Tricomi, Higher Transcendental Functions, Vol. III, McGraw-Hill, New York, 1955.

3. A. FrANSÉN, Addendum and Corrigendum to "High-precision values of the gamma function and of some related coefficients," Math. Comp., v. 37, 1981, pp. 233-235.

4. A. FransÉn \& S. WRIGGE, "High-precision values of the gamma function and of some related coefficients," Math. Comp., v. 34, 1980, pp. 553-566.

5. W. GAUTSCHI, "Polynomials orthogonal with respect to the reciprocal gamma function," BIT, v. 22 , 1982, pp. $387-389$.

6. G. H. Golub \& J. H. WelsCh, "Calculation of Gauss quadrature rules," Math. Comp., v. 23, 1969 , pp. 221-230, Microfiche supplement A1-A10.

7. S. A. GUSTAFSON, "Rapid computation of general interpolation formulas and mechanical quadrature rules," Comm. ACM, v. 14, 1971, pp. 797-801, Algorithm 417, p. 807.

8. G. H. Hardy, Ramanujan-Twelve Lectures on Subjects Suggested by His Life and Work, (reprinted), Chelsea, New York, 1959.

9. Collected papers of G. H. Hardy, Vols. I-VII (Especially Vol. IV, pp. 544-548), Oxford at the Clarendon Press, 1969.

10. W. A. Johnson, Private communication, 1982.

11. A. LindHAGEN, Studier öfver Gamma-Funktionen och Några Beslägtade Transcendenter (Studies of the gamma function and of some related transcendentals), Doctoral Thesis, B. Almqvist \& J. Wiksell's boktryckeri, Upsala, 1887.

12. W. F. Lunnon, P. A. B. Pleasants and N. M. Stephens, "Arithmetic properties of Bell numbers to a composite modulus I," Acta Arith., v. 35, 1979, pp. 1-16.

13. R. E. A. C. Paley \& N. Wiener, Fourier Transforms in the Complex Domain, Amer. Math. Soc., New York, 1934.

14. J. RIORDAN, Combinatorial Identities, Wiley, New York, 1968.

15. I. N. SNeddon, Special Functions of Mathematical Physics and Chemistry, Oliver and Boyd, Edinburgh and London, 1961.

16. S. WRIGGE, "A note on the moment generating function for the reciprocal gamma distribution," Math. Comp., v. 42, 1984, pp. 617-621.

17. M. WyMan \& R. WONG, "The asymptotic behaviour of $\mu(z, \beta, \alpha)$," Canad. J. Math., v. 21, 1969, pp. 1013-1023. 